\title{
Isolamento de Hisfoplasma capsulatum \\ do solo de Humboldt (Estado de Mato Grosso, Brasil)
}

\author{
Mário A. P. Moraes ( ${ }^{*}$ ) \\ Margarida M. R. Almeida ["]
}

\section{Resumo}

Histoplasma capsulatum foi isolado do solo de Humboldt, pequena localidade à margem do rio Aripuanã, no Estado de Mato Grosso. De seis amostras de terra, colhidas em quintais onde aves, principalmente galinhas, eram criadas, uma revelou-se positiva para o fungo. Em concordância com esse achado, um alto índice de positividade $(63,1 \%)$ ao teste da histoplasmina pôde ser observado entre os habitantes da vila.

\section{INTRODUÇÃo}

Até 1945, foi a infecção por Histoplasma capsulatum tida como rara e acidental. Efetivamente, em quase quatro décadas, período decorrido entre o descobrimento do fungo, por Darling (1906), e aquele ano, cerca de 50 casos apenas de histoplasmose, segundo Duarte (1945), haviam sido publicados.

Os trabalhos de Christie \& Peterson (1945) e Palmer (1945), mostraram, entretanto, que a histoplasmose, longe de ser, como se pensava, uma doença rara e fatal, é na verdade uma infecção quase sempre benigna, assintomática e muito freqüente em certas áreas geográficas. A maioria dos casos passa despercebida e a cura se faz espontaneamente. Esta forma benigna da infecção pode ser revelada pelo teste positivo à histoplasmina, ou pela presença de calciticações residuais nos pulmões. Em certas zonas endêmicas, como ocorre na parte central do Leste dos Estados Unidos, cerca de $80 \%$ da população são positivos à histoplasmina (Mochi \& Edwards, 1952).

Emmons et al. (1947), conseguiram isolar, pela primeira vez, $H$. capsulatum de roedores domésticos (Mus musculus e Rattus riorvegicus) capturados em uma comunidade rural, onde alguns casos humanos e caninos de his- toplasmose haviam sido encontrados. O achado, que se repetiu (Emmons, 1948; Emmons et al., 1949) em outras comunidades, sugeria a existência de um meio comum servindo de fonte de contágio ao homem e aos ratos. Isso levou Emmons (1949) a testar amostras de solo dos lugares onde viviam ratos infectados e, finalmente, $\in \mathrm{m}$ 1949, pôde ele referir o isolamento do fungo de 2 entre 387 amostras testadas.

A ocorrência de $H$. capsulatum no solo de comunidades rurais (fazendas, granjas etc.) está ligada, segundo mostraram Ajello et al. (1951) e Zeidberg et al. (1952), à presença na terra de excrementos de galinhas e outras aves. Posteriormente, reconheceu-se também a importância das fezes de morcegos (Emmons, 1958) ao serem descobertos casos agudos de histoplasmose em pessoas que haviam permanecido por algum tempo em grutas ou em outros locais freqüêntados por esses animais.

$\mathrm{Na}$ zona urbana, a fonte mais comum de infecção é o solo sob as árvores que servem de abrigo a pássaros. Emmons (1961), fez o primeiro isolamento de $H$. capsulatum do solo de uma área urbana, em Washington, D.C. As amostras de terra, colhidas junto às árvo res de uma zona comercial, estavam contaminadas por fezes de estorninhos, e todas as pessoas no local - compradores, visitantes $\theta$ vendedores - ficavam expostos à infecção através do pó levantado do chão pelo vento.

No Brasil, a distribuição de $H$. capsulatum no meio ambiente tem sido pouco estudada. Em 1959, Paula (1959) descreveu uma pequena epidemia de histoplasmose, envolvendo crianças de um internato, no Município de Paraíba do Sul, Rio de Janeiro. Acompanhadas de um professor, que também se infectou, essas crianças haviam estado em visita a umia

(") - Instituto Evandro Chagas da Fundaçāo Serviços de Saúde Pública, Belém. 
gruta antiga onde habitavam morcegos. Não se tentou o isolamento do fungo a partir do solo da mesma. Outra microepidemia, devida a fezes de morcego, que se derramaram do forro de uma casa, desta vez com isoiamento do fungo, ocorreu no litoral norte de São Paulo, e foi descrita por Fava Netto et. al. (1967)

Diretamente do solo, por meio de inoculação em camundongos, conseguiram Siıva (1956) e Araújo (1970) isolar H. capsulatum, respectivamente, na Bahia e em Minas Gerais Silva \& Paula (1956) também isolaram o fungo de ratos $(R$. norvegicus) capturados em Sal. vador, Bahia.

O achado acidental de ratos silvestres, pertencentes ao gênero Proechimys, infecta. dos naturalmente por $H$. capsulatum, em vários pontos da Amazônia (Lainson \& Shaw, 1975), veio mostrar ser bem freqüente a presença do mesmo no solo da região. Este tra balho descreve parte de uma investigação sistemática, que tem sido levada a efeito pelo Instituto Evandro Chagas, sobre histoplasmo. se, na Amazônia, e se refere ao isolamento de $H$. capsulatum do solo da vila de Humboldt, Estado do Mato Grosso.

\section{MATERIAL E MÉTODOS}

Em novembro de 1975, colheram-se amostras de solo dos quintais de seis residências da vila de Humboldt, à margem direita do rio Aripuanã, no Município do mesmo nome. A população, à época da coiheita, era aproximadamente de 600 pessoas.

Todas as casas selecionadas - as de $n^{\circ} \mathrm{s}$. $12,24,40,53,55$ e 68 (SUCAM) - possuiam galinheiros ou então aves criadas soltas no quintal.

Cada amostra, no laboratório em BelémPará, foi dividida em duas partes: uma para inoculação em camundongos e hamsters e outra para isolamento de ceratinófilos do solo. $\mathrm{Da}$ parte destinada à inoculação, uma pequena quantidade (cerca de 10 gramas) era suspensa em $90 \mathrm{ml}$ de água destilada estéril, em um frasco de Erlenmeyer de $100 \mathrm{ml}$ de capacidade. Após agitação vigorosa o frasco ficava em repouso, por uma hora, à temperatura ambiente. Do fluído sobrenadante, inoculava-se $1 \mathrm{ml}$ na ca- vidade peritoneal de camundongos e hamsters Para cada amostra utilizaram-se 6 camundongos e 2 hamsters. A inoculação dos camundongos ocorreu em janeiro e a dos hamsters $\mathrm{em}$ junho de 1976. Para o isolamento de fun gos ceratinófilos, empregou-se a técnica de Vanbreuseghem (1952) com cabelos humanos.

Os camundongos e hamsters foram sacrificados, respectivamente, 120 e 90 dias após a inoculação. Pedaços de fígado e baço eram então removidos e colocados em tubos com Sabouraud-glicose. Também se procedeu à pesquisa direta do parasito em impressões desses órgãos coradas pelo Giemsa.

\section{RESUltados}

Todos os camundongos inoculados com $\hat{\imath}$ terra das casas de $n^{\circ}$ s. 12, 40, 53, 55 e 68 foram negativos. Aqueles que receberam a terra dà casa de n. 24 apresentavam, ao serem sacrificados, aumento do fígado e do baço. As impressões destes órgãos, coradas pelo Giemsa, mostraram numerosos corpúsculos arredondados ou ovais, medindo 2 a 3 ; , Je diâmetro, iniracelulares, que foram identificados como a forma leveduriforme de $H$. capsulatum. Os fragmentos da semeadura em Sabouraud-glicose deram nascimento, ao fim de 5 dias, com os tubos mantidos à temperatura ambiente $\left(26-28^{\circ} \mathrm{C}\right)$, a colônias brancas, penugentas, que com o tempo iam adquirindo uma tonalidade creme. O achado, posteriormente, dos macroconídios característicos, de superfície espinhosa (estalagmósporos) con. firmou o diagnóstico da espécie.

Cerca de dois meses após o sacrifício aos camundongos, procedieu-se à inoculação dos hamsters, em princípio para verificar-se o comportamento das demais amostras, sabido que os hamsters são mais sensíveis ao $H$. capsu. latum do que os camundongos. As amostras de terra, acondicionadas em sacos de plástico, já estavam no laboratório há mais de seis meses, à temperatura da sala. Os resultados, apesar do prazo decorrido, foram idênticos aos da primeira inoculação: apenas os hamsters inoculados com a amostra da casa n. 24 adquiriram a doença. Tal como da primeira vez, $H$. capsulatum foi obtido em cultura a partir das vísceras. 
Três espécies de fungos ceratinofílicos pu. deram ser isolados das seis amostras Chrysosporium indicum, Chrysosporium tropicum e Arthroderma tuberculatum. O primeiro estava presente no solo das casas de n's. 24, $40,53,55$ e 68 . C. tropicum e A. tuberculatum, apenas no solo da casa de $n .^{\circ} 12$.

\section{COMENTÁRIOS}

Poucos têm sido os casos de histoplasmose-doença descritos na região amazônica. Em revisão feita, até o ano de 1972, encontrou Lobão (1972) referência a apenas dois casos no Estado do Pará. Um deles era proveniente do Estado do Amazonas. Recentemente, en- contramos em Belém um novo caso, o qual procedia da localidade Marituba, Município de Ananindeua. Até onde é de nosso conhecimento, nenhum caso foi registrado no Estado do Amazonas.

Apesar disso, todos os inquéritos com histoplasmina, levados a cabo em diferentes pontos da Amazônia, têm acusado sempre uma alta proporção de reatores, o que indica ser a histoplasmo-infecção bem freqüente na área. Costa \& Cerqueira (1965), em crianças de idade entre 6 e 12 anos, encontraram 12,5\% de positivos, na cidade de Belém. Nesta mesma cidade, Tesh \& Marques (1966), em 258 adultos (34 estudantes de medicina e 224 pacientes) obtiveram $43,4 \%$ de reatores. Na cidade
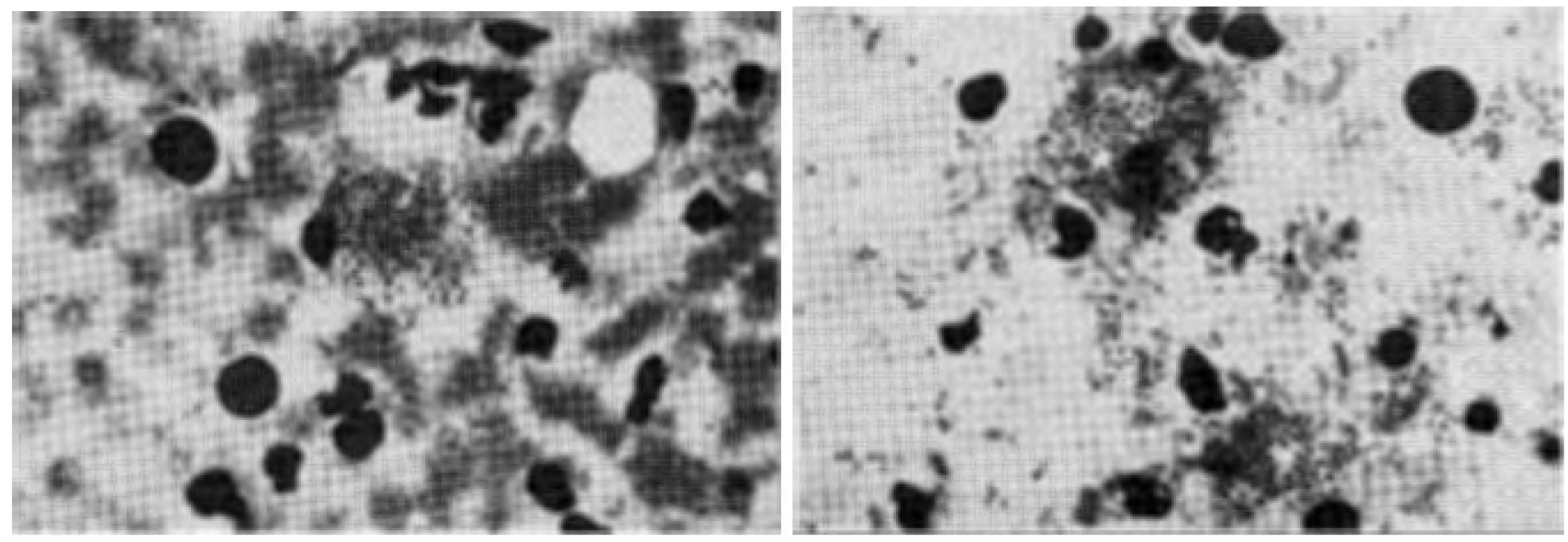

Fotos 1 e 2 - Histiócitos contendo numerosas formas em levedo de Histoplasma capsulatum. Impressão de fígado de hamster. $800 \mathrm{x}$. Giemsa.
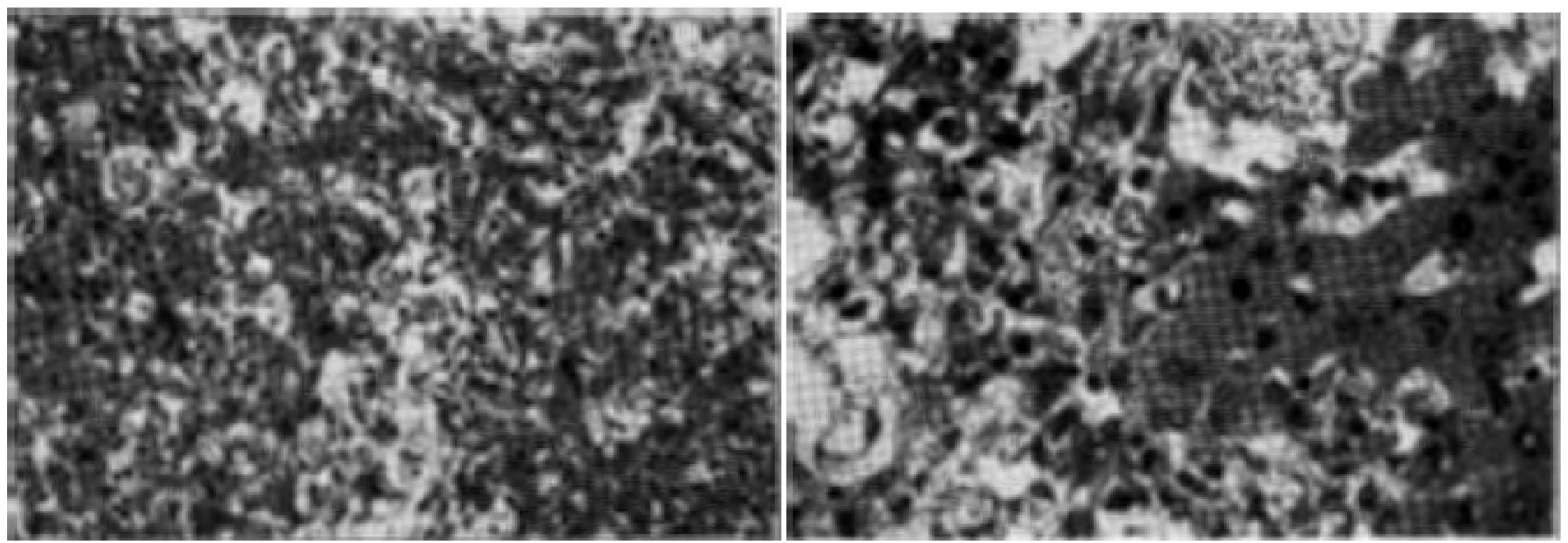

Fotos 3 e 4 - Corte de fígado de hamster. As células de Küpffer, volumosas, contêm numerosos corpúsculos arredondados de $H$. capsulatum. 160 e $400 x$. H.E. 
de Santarém, dentre 623 escolares testados por Morehead \& Macedo (Apud Mochi \& Edwards, 1952), $30,2 \%$ mostraram-se positivos ao teste intradérmico. Dentro ainda do Estado do Pará, Lacaz et al. (1967) encontraram 27,4\% de reatores em Conceição do Araguaia, e Boulos et al. (1975), índices, respectivamente, de $25,7 \%$ e $32,5 \%$, nas localidades de Itupiranga e São João do Araguaia.

Percentuais bem altos foram também encontrados por Fonseca et al. (1973) no Estado do Amazonas, especialmente em Tabatinga $(44 \%)$ e em Coari e Tefé $(69 \%)$. Ao todo, dentre 294 pessoas testadas, $40 \%$ exibiram reação positiva.

$\mathrm{Na}$ mesma época em que se coletaram as amostras de solo, um inquérito intradérmico com histoplasmina, compreendendo 95 pessoas, teve lugar em Humboldt, feito pelo Instituto Evandro Chagas. Considerando como positivos ao teste apenas os indivíduos nos quais houve a formação de uma pápula eritematosa com mais de $5 \mathrm{~mm}, 63,1 \%$ dos 95 habitantes reagiram positivamente à histoplasmina. A distribuição por sexo e idade dos reatores pode ser vista no Quadro I. É de ressaltar-so que todos esses indivíduos procediam de outras localidades do Estado, ou então do Amazonas e Acre.

O isolamento, relativamente fácil, de $H$. capsulatum do solo de Humboldt demonstra a grande freqüência deste no local, o que explica o alto índice de positividade ao teste da histoplasmina verificado.

\section{QUADRO I}

TESTE INTRADERMICO DE HISTOPLASMINA EM HABITANTES DE HUMBOLDT (MATO GROSSO. BRASIL) POR SEXO E IDADE

\begin{tabular}{crrrrrrrrr}
\hline & & H O M E N S & \multicolumn{3}{c}{ MULHERES } & \multicolumn{3}{c}{ AMBOS OS SEXOS } \\
\hline IDADE & N.0 & + & $\%$ & N.0 & + & $\%$ & N.0 & + & $\%$ \\
$0-9$ & 0 & 0 & 0 & 0 & 0 & 0 & 0 & 0 & 0 \\
$10-19$ & 7 & 5 & 71 & 8 & 4 & 50 & 15 & 9 & 60 \\
$20-29$ & 19 & 12 & 63 & 15 & 12 & 80 & 34 & 24 & 70 \\
$30-39$ & 13 & 7 & 53 & 7 & 5 & 71 & 20 & 12 & 60 \\
$40-49$ & 9 & 6 & 66 & 3 & 2 & 66 & 12 & 8 & 66 \\
50 e + & 7 & 3 & 42 & 7 & 4 & 57 & 14 & 7 & 50 \\
\hline Total & 55 & 33 & 60 & 40 & 27 & 67 & 95 & 60 & 63 \\
\hline
\end{tabular}

\section{SUMMARY}

Histoplasma capsulatum was isolated from the soil of Humboldt, a small village on the Aripuanã River, State of Mato Grosso. Of six samples collected in the backyards of houses, where domestic fowls were reared, one was positive for this fungus. In accordance with this finding, a high percentage $(63,1 \%)$ of positive reactions to the intradermal test of histoplasmin was observed among the inhabitants of Humboldt.

\section{BIBLIOGRAFIA CITADA}

AJello, L. \& ZeIDBERG, L.D.

1951 - Isolation of Histoplasma capsulatum and Allescheria boydii from soil. Science $113: 662-663$.
AraúJio, F.G.

1970 - Primeiro isolamento de Histoplasma capsulatum de solo em Minas Gerais. Rev. Inst. Med. trop. São Paulo 12 : 185-191.

Boulos, M.; PINTo, W.P.; Labonia Fo., W.; Lancarotte, I.; Draibe, S.E.; Shiroma, M. \& Fava NetTo, C.

1975 - Inquérito imuno-alérgico com paracoccidioidina e histoplasmina nas localidades de Itupiranga e de São João do Araguaia, Pará. Comunicação apresentada no XI Congresso da Sociedade Brasileira de Medicina Tropical (Rio de Janeiro), 23-28 fevereiro. 
Christie, A. \& Peterson, J.C.

1945 - Pulmonary calcification in negatve reactors to tuberculin. Amer. J. Publ. Health $35: 1131-1147$.

Costa, C.A. \& Cerqueira, R.J

1965 - Primeiras pesquisas sobre o teste intradérmico da histoplasmina num grupo de crianças de Belém-Pará. Rev. Ciências Biológicas (Univ. Fed. Pará) $3: 31-33$.

DARLING, S.T.

1906 - A protozoon general infection producing pseudotubercles in the lungs, and focal necrosis in the liver, spleen and lymph nodes. J. Amer. Med. Assoc. 46:1283-1235.

DuARTE, E.

1945 - Histoplasmose. Memórias do Inst. Oswaldo Cruz $43: 457-494$.

Emmons, C.W.; Bell, J.A. \& Olson, B.J.

1947 - Naturally occurring histoplasmosis in Mus musculus e Rattus norvegicus. Pub. Health Rep. 62 : 1642-1646.

Emmons, C.W.

1948 - Histoplasmosis in wild rats; occurrence and histopathology. Pub. Health Rep. $63: 1416-1422$

Emmons, C.W.; Morlan, H.B. \& Hill, E.L.

1949 - Histoplasmosis in rats and skunks in Georgia. Pub. Health Rep. 64 : 1423-1430.

EMmoNs, C.W

1949 - Isolation of Histoplasma capsulatum from soil. Pub. Health Rep, 64 : 892-896.

EMMONS, C.W.

1958 - Association of bats with histoplasmosis. Pub. Health Rep. $73: 590-595$.

EMMONS, C.W.

1961 - Isolation of Histoplasma capsulatum from soil in Washington, D.C. Pub. Health Rep. 76 : 591-596.

Faya Netto, C.; Andrade e Silva, U.; Chammas, F. \& LACAZ, C.S.

1967 - Histoplasmose epidêmica. Estudo clínico, radiológico, micológico e imunológico de surto ocorrido no Estado de São Paulo. Rev. Inst. Med. trop. São Paulo 9 : 222-232.

Fonseca, O.J.M.; LaCaz, C.S. \& Machado, P.A.

1973 - Inquérito imuno-alérgico na Amazônia. Resultados preliminares. Rev. Inst. Med. trop. São Paulo 15 : 409-416.
LacaZ, C.S.; Padim, M.V. \& Minami, P.S

1967 - Reações à histoplasmina em dois povoados brasileiros: Arraias (Estado de Goiás) e Conceição do Araguaia (Estado do Pará). Hospital $71: 87-90$.

LAINSON, R. \& SHAW, J.J

1975 - Pneumocystis and Histoplasma infections in wild animals from the Amazon Region of Brazil. Trans. Roy. Soc. Trop. Med. Hyg. $69: 505-508$.

LOBÃo, A.C.

1972 - Histoplasmose; aspectos relativos ao Estado do Pará. Rev. Univ. Fed. Pará 2 (Série 2) : 305-351.

MochI, A. \& Edwards, P.Q.

1952 - Geographical distribution of histoplasmosis and histoplasmin sensitivity. Bull. World Health Org. 5 : 259-291.

Palmer, C.E.

1945 - Nontuberculous pulmonary calcification and sensitivity to histoplasmin. Pub. Health Rep. 60 : 513-520.

Paula, A

1959 - Microepidemia de histoplasmose. Rev. Serv. Nac. Tuberculose $3: 11-20$.

Silva, M.E. \& Paula, L.A.

1956 - Infecção natural de ratos pelo Histoplasma capsulatum na cidade de Salvador, Bahia. Bol. Fund. Gonçalo $\mathbf{M u}$. niz, N. $\circ 9$.

SILVA, M.E.

1956 - Isolamento de Histoplasma capsulatum do solo de zona endêmica de calazar na Bahia. Bol. Fund. Gonçalo Muniz $10: 1-15$

TeSh, R.B. \& Marques, R.J.

1966 - Histoplasmin sensitivity in Brazil. Report of recent skin test survey in Recife (Pernambuco) and Belém (Pará) and review of other surveys in various areas of the country. Amer. J. Trop. Med. Hyg. $15: 359-363$.

VANBREUSEGHEM, $\mathbf{R}$.

1952 - Technique biologique pour l'isolement des dermatophytes du sol. Ann. Soc. Belge Med. Trop. $32:$ 173-178.

Zeidberg, L.D.; A.Jello, L.; Dillon, A. \& Runyon, L.C. 1952 - Isolation of Histoplasma capsulatum from soil. Amer. J. Publ. Health 42: 930-935. 